\title{
Numerical solution of inverse source problem for one-dimensional integer/fractional order diffusion equation by one point observation data
}

\author{
Sen Zhang, Zhousheng Ruan
}

\begin{abstract}
In this paper, we consider reconstructing the space-dependent source of a one-dimensional fractional diffusion equation by observing the data from the left endpoint. First, we analyze the ill-posedness of the problem, and then use the Laplace transform and analytical continuation techniques to prove the uniqueness of the inverse source problem. Then, the inverse source problem is transformed into a variational optimization problem by Tikhonov regularization method. The gradient of the functional is derived based on the idea of variational adjoint method, and then the conjugate gradient method is used to solve the problem. Finally, we give several numerical examples to show the effectiveness of the proposed method.
\end{abstract}

Index Terms - conjugate gradient algorithm, inverse source problem, Laplace transform, Tikhonov regularization, uniqueness

\section{INTRODUCTION}

In recent years, many researchers have conducted a lot of research on fractional diffusion equations. However, for some practical problems, the initial conditions of the research object, partial boundary data or source terms are unknown, and we hope to reconstruct them by additional observation data, which leads to the inverse problem of the fractional diffusion equation [1]-[6].Therefore, the research on the inverse problem of fractional diffusion equation has very important practical significance.

There are a lot of valuable research results in the inverse problem of fractional diffusion equations. In [7], Xiong and Xue proposed a new fractional Tikhonov method to solve an inverse source problem for the time-fractional diffusion equation with variable coefficients in a general bounded domain. Yan and Wei consider an inverse space-dependent source problem for a time-fractional diffusion equation by an adjoint problem approach, in [8]. In [9],Wang, Zhang and Wu turns the inversion source problem into a regular optimization problem, and then proposes a non-iterative algorithm based on a sequence well-posed direct problems solved by the finite element method is proposed for solving the optimization problem. Ruan, Yang and Lu reconstructs the inverse problem of time fractional diffusion equation into an operator equation by Fourier method, and then proposes a classical Tikhonov regularization method to solve this problem in paper [10]. In order to overcome the ill-posedness of the inverse problem of the diffusion equation, Wang and Wei proposed a quasi-reversibility method in [11]. There is too much research on the inverse problem of diffusion

First Author name, School of Science, East China University of Technology, Nanchang, Jiangxi, 330013.

Second Author name, School of Science, East China University of Technology, Nanchang, Jiangxi, 330013. equations [12]-[19], but the above mentioned interesting references of inverse problems for diffusion equation uniformly take the final observation as the additional data. To our knowledge, there is relatively little research on the inverse problem for the diffusion equation with the local observation data. We consider the following diffusion equation in this paper

$\frac{\partial^{\alpha} u(x, t)}{\partial t^{\alpha}}=(L u)(x, t)+f(x), \quad x \in(0, l), t>0,0<\alpha \leq 1$

with homogeneous Neumann boundary condition

$\frac{\partial u}{\partial x}(0, t)=0, \frac{\partial u}{\partial x}(l, t)=0, t \geq 0$,

and nonhomogeneous initial condition

$u(x, 0)=\varphi(x), \quad x \in[0, l]$,

The following is the definition of the symmetric uniformly elliptic operator $-L$

$-L(u)=-\frac{\partial}{\partial x}\left(a(x) \frac{\partial}{\partial x} u(x, t)\right)+c(x) u(x, t)$,

$a(x) \in C^{1}[0, l], c(x) \in C[0, l], c(x) \geq 0, \forall x \in[0, l]$, and there exists a constant $v>0$, such that $a(x)>v, \forall x \in[0, l]$. Here $\partial_{t}^{\alpha}$ denotes the Caputo fractional derivative with respect to $t$ and is defined by

$\frac{\partial^{\alpha} f(t)}{\partial t^{\alpha}}= \begin{cases}\frac{1}{\Gamma(1-\alpha)} \int_{0}^{t} f^{\prime}(\tau) d \tau, & t>0,0<\alpha<1, \\ \frac{d f(t)}{d t}, & t>0, \alpha=1 .\end{cases}$

We consider the observation data from the left endpoint to identify the space-dependent source

$g(t)=u[f](0, t), t \in[0, T]$.

In general, measuring error is inevitable, the observation data $g(t)$ contain measurement errors and satisfies

$\square g^{\delta}(\cdot)-g(\cdot) \square_{L^{2}(0, T)} \leq \delta$

where $\delta$ is the error level.

As the initial-boundary value problem (1)-(3) is a linear system, the principle of superposition is valid. Without prejudice to the generality, for the sake of research convenience, we assume the initial condition is homogeneous, i.e., $\varphi(x)=0$. We organize the rest of the paper as follows, in section 2, introduce some preliminary knowledge and analyze the ill-posedness of the inversion source problem. We prove the uniqueness of the inversion source problem in section 3. In section 4, we transform the inversion source problem into a Tikhonov regularization optimization problem, and the conjugate gradient algorithm is proposed based on the variational adjoint technique. Finally, we give some numerical examples in section 5 . 


\section{PRELIMINARIES AND ILL-POSEDNESS ANALYSIS}

\section{A. Preliminaries}

Throughout this article, we use the following definitions and lemmas given in [20] and [21].

Definition 1. The definition of the two-parameters Mittag Leffler functions is as follows

$$
E_{\alpha, \beta}(z)=\sum_{k=0}^{\infty} \frac{z^{k}}{\Gamma(\alpha k+\beta)}, z \in \square
$$

where $\alpha>0, \beta>0$ are arbitrary constants.

Lemma 1. (1) Let $0<\alpha<1, \beta \in \square$ be arbitrary constants. We suppose that $\bar{\mu}$ is such that $\frac{\alpha}{2} \pi<\bar{\mu}<\{\pi, \pi \alpha\}$. There has a constant $c_{+}>0$ such that

$$
\left|E_{\alpha, \beta}(z)\right| \leq \frac{c_{+}}{1+|z|}, \bar{\mu} \leq|\arg (z)| \leq \pi .
$$

(2) Let $\lambda>0,0<\alpha<1$, we have $\partial_{0_{+}}^{\alpha} E_{\alpha, 1}\left(-\lambda t^{\alpha}\right)=-\lambda E_{\alpha, 1}\left(-\lambda t^{\alpha}\right)$, $\frac{d}{d t} E_{\alpha, 1}\left(-\lambda t^{\alpha}\right)=-\lambda t^{\alpha-1} E_{\alpha, \alpha}\left(-\lambda t^{\alpha}\right), t>0$.

(3) Let $0<\alpha<1$, then we have $0<E_{\alpha, 1}(-t)<1$ with any $\mathrm{t}>0$. Moreover, $E_{\alpha, 1}(-t)$ is completely monotonic function, i.e.,

$$
(-1)^{n} \frac{d^{n}}{d t^{n}} E_{\alpha, 1}(-t) \geq 0, \forall n \in \square
$$

As the differentiation operator $-L$ is a symmetric uniformly elliptic operator, the spectrum of $-L$ only includes the eigenvalues which are counting according to the multiplicities. Let $\left\{\lambda_{k}, \phi_{k}(x)\right\}, k=1, \cdots$, be the eigensystem of $-L$. Here we take $\phi_{k}$ satisfying $\phi_{k}(0)=1$ and set $\tilde{\mathrm{n}}_{k}=\left\|\phi_{k}(x)\right\|_{L^{2}(\Omega)}^{-2}, k=1, \cdots$. From [22], we see that $\tilde{\mathrm{n}}_{k}=c_{0}+o(1)$.

We know that the sequence $\left\{\phi_{k}(x)\right\}_{k=0}^{\infty}$ forms an orthonormal basis in $L^{2}(0, l)$. We define a fractional power $(-L)^{\gamma}$ of $-L$ as follow

$$
D\left((-L)^{\gamma}\right)=\left\{\psi: \sum_{k=1}^{\infty} \lambda_{k}^{2 \gamma} \rho_{k}\left|<\psi, \phi_{k}>\right|^{2}<\infty\right\},
$$

where $\langle\cdot, \cdot\rangle$ is the scalar product in $L^{2}(0, l)$. We know that $D\left((-L)^{\gamma}\right)$ is a Hilbert space with the following norm:

$$
\|\psi\|_{D\left((-L)^{\gamma}\right)}=\left\{\sum_{k=1}^{\infty} \lambda_{k}^{2 \gamma} \rho_{k}\left|<\psi, \phi_{k}>\right|^{2}\right\}^{\frac{1}{2}} .
$$

We have $D\left((-L)^{\gamma}\right) \subset L^{2}(0, l)$ for $\gamma \geq 0$.

In order to analyze the ill-posedness of this problem, we define the weak solution to system (1)-(3) as follows.

Definition 2. Let $f(x) \in L^{2}(0, l), u$ is a weak solution to the initial-boundary value problem (1)-(3) with $\varphi=0$ if $u \in C\left(0, T ; D\left((-L)^{\alpha}\right)\right)$.

Lemma 2. Assume $f(x) \in L^{2}(0, l)$, then the direct problem (1)-(3) has a unique weak solution expressed by series expression and has the following estimate

$\|u(\cdot, t)\|_{D\left((-L)^{\alpha}\right)}+\left\|\partial_{t}^{\alpha} u(\cdot, t)\right\|_{L^{2}(\Omega)} \leq C t^{-\alpha}\|f\|_{L^{2}(0, l)}, 0<\alpha \leq 1$.

Proof . The application variable separation method expresses the series solution of the direct problem (1)-(3) as follows

$$
u(x, t)= \begin{cases}\sum_{k=1}^{\infty} \frac{f_{k}}{\lambda_{k}} \rho_{k}\left(1-E_{\alpha, 1}\left(-\lambda_{k} t^{\alpha}\right)\right) \phi_{k}(x), & x \in[0, l], t \geq 0,0<\alpha<1, \\ \left.\sum_{k=1}^{\infty} \frac{f_{k}}{\lambda_{k}} \rho_{k}\left(1-e^{-\lambda_{k} t}\right)\right) \phi_{k}(x), & t \geq 0, \alpha=1 .\end{cases}
$$

where $f_{k}=<f(x), \phi_{k}(x)>, k=1, \cdots, \infty$. We have

$$
\begin{aligned}
\|u(\cdot, t)\|_{D\left((-L)^{\alpha}\right)} & = \begin{cases}\left\|\sum_{k=1}^{\infty} \frac{f_{k}}{\lambda_{k}} \rho_{k}\left(1-E_{\alpha, 1}\left(-\lambda_{k} t^{\alpha}\right)\right) \phi_{k}(\cdot)\right\|_{D\left((-L)^{\alpha}\right)}, & t>0,0<\alpha<1, \\
\left.\| \sum_{k=1}^{\infty} \frac{f_{k}}{\lambda_{k}} \rho_{k}\left(1-e^{-\lambda_{k} t}\right)\right) \phi_{k}(\cdot) \|_{D\left((-L)^{\alpha}\right)}, & t>0, \alpha=1 .\end{cases} \\
& \leq \sum_{k=1}^{\infty}\left\|\frac{f_{k}}{\lambda_{k}} \rho_{k} \phi_{k}(\cdot)\right\|_{D\left((-L)^{\alpha}\right)}, 0<\alpha \leq 1, \\
& \leq C t^{-\alpha}\|f\|_{L^{2}(0, l)}, t>0,0<\alpha \leq 1 .
\end{aligned}
$$

Similarly, we have

$$
\begin{aligned}
\left\|\partial_{t}^{\alpha} u(\cdot, t)\right\|_{L^{2}(0, l)} & = \begin{cases}\left\|\sum_{k=1}^{\infty} \frac{f_{k}}{\lambda_{k}} \rho_{k}\left(1-E_{\alpha, 1}\left(-\lambda_{k} t^{\alpha}\right)\right) \phi_{k}(\cdot)\right\|_{L^{2}(0, l)}, & t>0,0<\alpha<1, \\
\left\|\sum_{k=1}^{\infty} \frac{f_{k}}{\lambda_{k}} \rho_{k}\left(1-e^{-\lambda_{k} t}\right) \phi_{k}(\cdot)\right\|_{L^{2}(0, l)}, & t>0, \alpha=1 .\end{cases} \\
& \leq C t^{-\alpha}\|f\|_{L^{2}(0, l)}, t>0,0<\alpha \leq 1 .
\end{aligned}
$$

Through the above definitions and proofs, we know that (2.4) is a weak solution of equations (1)-(3).

\section{B. Ill-posedness Analysis}

In order to prove the ill-posedness of the inverse problem, we define a linear operator $K_{\alpha}: L^{2}(0, l) \rightarrow L^{2}(0, T)$ as follows:

$$
K_{\alpha} f(x)= \begin{cases}\sum_{k=1}^{\infty} \frac{f_{k}}{\lambda_{k}} \rho_{k}\left(1-E_{\alpha, 1}\left(-\lambda_{k} t^{\alpha}\right)\right), & t \in(0, T), 0<\alpha<1, \\ \sum_{k=1}^{\infty} \frac{f_{k}}{\lambda_{k}} \rho_{k}\left(1-e^{-\lambda_{k} t}\right), & t \in(0, T), \alpha=1 .\end{cases}
$$

Theorem 1.

The observation operator $K_{\alpha}: f(x) \in L^{2}(0, l) \mapsto g(t) \in L^{2}(0, T)$ is compact.

Proof . In order to prove that $K_{\alpha}$ is a compact operator, we define the finite dimensional operator $K_{\alpha, N}$ as follows:

$$
K_{\alpha, N} f(x)= \begin{cases}\sum_{k=1}^{N} \frac{f_{k}}{\lambda_{k}} \rho_{k}\left(1-E_{\alpha, 1}\left(-\lambda_{k} t^{\alpha}\right)\right), & t \in(0, T), 0<\alpha<1, \\ \sum_{k=1}^{N} \frac{f_{k}}{\lambda_{k}} \rho_{k}\left(1-e^{-\lambda_{k} t}\right), & t \in(0, T), \alpha=1 .\end{cases}
$$

Then, from (2.7) and (2.8) and lemma 1, we obtain

$$
\begin{aligned}
\left\|K_{\alpha} f(x)-K_{\alpha, N} f(x)\right\|_{L^{2}(0, T)} & = \begin{cases}\left\|\sum_{k=N+1}^{\infty} \frac{f_{k}}{\lambda_{k}} \rho_{k}\left(1-E_{\alpha, 1}\left(-\lambda_{k} t^{\alpha}\right)\right)\right\|_{L^{2}(0, T)}, & 0<\alpha<1, \\
\left\|\sum_{k=N+1}^{\infty} \frac{f_{k}}{\lambda_{k}} \rho_{k}\left(1-e^{-\lambda_{k} t}\right)\right\|_{L^{2}(0, T)}, & \alpha=1,\end{cases} \\
& \leq \sum_{k=N+1}^{\infty}\left|\frac{f_{k}}{\lambda_{k}} \rho_{k}\right| \sqrt{T}, 0<\alpha \leq 1 .
\end{aligned}
$$

By $\lambda_{k} k^{2}$, we know $\left\|K_{\alpha, N}-K_{\alpha}\right\| \rightarrow 0$ in the sense of operator norm in $L^{2}(\Omega)$ as $N \rightarrow \infty$. Therefore, the observation operator $K_{\alpha}$ is compact.

We know that use the observation data $g(t)$ in $L^{2}(0, t)$ to identify space-dependent source $f(x)$ in $L^{2}(0, l)$ is ill-posed, need to consider some regularization methods. Therefore, we consider solving the inverse source problem by the Tikhonov regularization method in section 4 .

\section{UNIQUENESS}

In order to explore the unique theorem of the inverse source problem, first we need to prove the following lemmas. Lemma 3. Let $f(x) \in L^{2}(0, L)$, the direct problem (1)-(3) exist a unique solution $u(x, t)$. Moreover, we have the following estimate

$\|u\|_{L^{2}\left(0, T ; H^{2}(0, l)\right)} \leq C\|f\|_{L^{2}(0, l)}$, 
and

$\|u\|_{C[0, T ; C[0, l]]} \leq C\|f\|_{L^{2}(0, l)}$.

Proof . From the weak solution, we obtain

$$
\begin{aligned}
& \|u\|_{L^{2}\left(0, T ; H^{2}(0, l)\right)} \\
& =\left\{\begin{array}{l}
\left\|\sum_{k=1}^{\infty} \frac{f_{k}}{\lambda_{k}} \rho_{k}\left(1-E_{\alpha, 1}\left(-\lambda_{k} t^{\alpha}\right)\right) \phi_{k}(\cdot)\right\|_{L^{2}\left(0, T ; H^{2}(0, l)\right)}, 0<\alpha<1, \\
\left\|\sum_{k=1}^{\infty} \frac{f_{k}}{\lambda_{k}} \rho_{k}\left(1-e^{-\lambda_{k} t}\right) \phi_{k}(\cdot)\right\|_{L^{2}\left(0, T ; H^{2}(0, l)\right)}, \alpha=1,
\end{array}\right. \\
& \leq\left\{\begin{array}{l}
\left(\sum_{k=1}^{\infty} \lambda_{k}^{4}\left(\frac{f_{k}}{\lambda_{k}}\right)^{2} \rho_{k}\left(1-E_{\alpha, 1}\left(-\lambda_{k} t^{\alpha}\right)\right)^{2} d t\right)^{\frac{1}{2}}, 0<\alpha<1, \\
\left(\sum_{k=1}^{\infty} \lambda_{k}^{4}\left(\frac{f_{k}}{\lambda_{k}}\right)^{2} \rho_{k}\left(1-e^{-\lambda_{k} t}\right)^{2} d t\right)^{\frac{1}{2}}, \alpha=1,
\end{array}\right. \\
& \leq\left(\sum_{k=1}^{\infty} \lambda_{k}^{2} f_{k}^{2} \rho_{k} T\right)^{\frac{1}{2}} \leq C\|f\|_{L^{2}(0, l)}, 0<\alpha \leq 1 .
\end{aligned}
$$

By the Sobolev embedding theorem, we get

$$
\left\|\phi_{k}\right\|_{C[0, l]} \leq \tilde{C} \lambda_{k}^{\frac{1}{4+\grave{o}}}\left\|\phi_{k}\right\|_{L^{2}[0, l]}, \forall \grave{o}>0 .
$$

Note that $\left\|\phi_{k}\right\|_{L^{2}[0, l]}=O\left(\frac{1}{\sqrt{c_{0}}}\right)$. We have

$$
\begin{aligned}
& \max _{x \in[0, l]}|u(x, t)| \\
& =\left\{\begin{array}{cc}
\max _{x \in[0, l]}\left|\sum_{k=1}^{\infty} \frac{f_{k}}{\lambda_{k}} \rho_{k}\left(1-E_{\alpha, 1}\left(-\lambda_{k} t^{\alpha}\right)\right) \phi_{k}(x)\right| & , 0<\alpha<1, \\
\max _{x \in[0, l]}\left|\sum_{k=1}^{\infty} \frac{f_{k}}{\lambda_{k}} \rho_{k}\left(1-e^{-\lambda_{k} t}\right) \phi_{k}(x)\right| & , \alpha=1,
\end{array}\right. \\
& \leq\left\{\begin{array}{c}
\tilde{C}\left|\frac{f_{k}}{\lambda_{k}}\right| \rho_{k}\left(1-E_{\alpha, 1}\left(-\lambda_{k} t^{\alpha}\right)\right) \lambda^{\frac{1}{4}+\dot{o}}, 0<\alpha<1, \\
\tilde{C}\left|\frac{f_{k}}{\lambda_{k}}\right| \rho_{k}\left(1-e^{-\lambda_{k} t}\right) \lambda^{\frac{1}{4}+\grave{o}}, \alpha=1, \\
\leq \tilde{C} \sum_{k=1}^{\infty} \rho_{k} \lambda_{k}\left|\frac{f_{k}}{\lambda_{k}}\right| \lambda_{k}^{\frac{1}{4}+\dot{\mathrm{o}}-1} \leq \tilde{C}\|f\|_{L^{2}(0, l)} \sqrt{\sum_{k=1}^{\infty} \lambda^{-\frac{3}{2}+2 \dot{\mathrm{o}}}}
\end{array}\right. \\
& \leq C|| f \|_{L^{2}(0, l)}, \forall t \geq 0 .
\end{aligned}
$$

From the above inequality, we have $\|u\|_{C[0, T ; C[0, l]]} \leq C\|f\|_{L^{2}(0, l)}$.

Lemma 4. Assume $f(x) \in L^{2}(0, l)$, then the source $f(x)$ can be identified uniquely by the observation data at left end point, i.e., $g(t)=u(0, t), t \in[0, T]$.

Proof . By contradiction, we assume there exist two different functions, denoting by $f_{i}(x), i=1,2$. The solutions relative to $f_{1}(x)$ and $f_{2}(x)$ are expressed as follows

$u_{i}(x, t)= \begin{cases}\sum_{k=1}^{\infty} \frac{f_{i, k}}{\lambda_{k}} \rho_{k}\left(1-E_{\alpha, 1}\left(-\lambda_{k} t^{\alpha}\right)\right) \phi_{k}(x), & x \in[0, l], t \in[0, T], 0<\alpha<1, \\ \sum_{k=1}^{\infty} \frac{f_{i, k}}{\lambda_{k}} \rho_{k}\left(1-e^{-\lambda_{k} t}\right) \phi_{k}(x), & x \in[0, l], t \in[0, T], \alpha=1 .\end{cases}$

Where $f_{i, k}=<f_{i}(x), \phi_{k}(x)>, i=1,2, k=1, \cdots, \infty$. For the sake of convenience, we only consider the case of $0<\alpha<1$ in detail. By lemma 3, the two series $\sum_{k=1}^{\infty} \frac{f_{1, k}}{\lambda_{k}} \rho_{k}\left(1-E_{\alpha, 1}\left(-\lambda_{k} t^{\alpha}\right)\right)$ and $\sum_{k=1}^{\infty} \frac{f_{2, k}}{\lambda_{k}} \rho_{k}\left(1-E_{\alpha, 1}\left(-\lambda_{k} t^{\alpha}\right)\right)$ are uniformly convergent on interval $[0, T]$. By analytic continuation technique, we know that the above two series are uniform convergence on $[0, \infty)$. By lemma 3, have

$\left|e^{-t R e z} \sum_{k=1}^{\infty} \frac{f_{i, k}}{\lambda_{k}}\left(1-E_{\alpha, 1}\left(-\lambda_{k} t^{\alpha}\right)\right) \rho_{k}\right| \leq e^{-t R e z}\left\|f_{i}\right\|_{L^{2}(0, l)}, t>0, i=1,2$.

As $e^{-t R e z}$ is integral in $t \in(0, \infty)$ for fixed $z$ such that Rez $>0$. Taking Laplace transform with respect to the time variable $t$ on the both sides of equation $u_{1}(0, t)=u_{2}(0, t)$, by the Lebesgue dominated convergence theorem, we have

$\sum_{k=1}^{\infty} \frac{f_{1, k}}{\lambda_{k}} \rho_{k} \frac{1}{z^{\alpha}+\lambda_{k}}=\sum_{k=1}^{\infty} \frac{f_{2, k}}{\lambda_{k}} \rho_{k} \frac{1}{z^{\alpha}+\lambda_{k}}, \operatorname{Rez}>0$.

That is

$\sum_{k=1}^{\infty} \frac{f_{1, k}}{\lambda_{k}} \rho_{k} \frac{1}{\eta+\lambda_{k}}=\sum_{k=1}^{\infty} \frac{f_{2, k}}{\lambda_{k}} \rho_{k} \frac{1}{\eta+\lambda_{k}}$, Re $>0$.

We know that $\sum_{k=1}^{\infty} \frac{f_{i, k}}{\lambda_{k}} \rho_{k} \frac{1}{\eta+\lambda_{k}}$ is internally closed uniform convergence in $\eta \in \square V \mid\left\{-\lambda_{k}\right\}_{k=1}^{\infty}$. So, applying the Weierstrass theorem, we can analytically continue $\sum_{k=1}^{\infty} \frac{f_{i, k}}{\lambda_{k}} \rho_{k} \frac{1}{\eta+\lambda_{k}}$ in $\eta$ and (22) holds for $\eta \in \square \backslash \mid \|\left\{-\lambda_{k}\right\}_{k=1}^{\infty}$ We take a suitable disk which only includes $-\lambda_{1}$ and does not include others. Integrating (21) in the disk, we get $2 \pi \rho_{1} f_{1,1}=2 \pi \rho_{1} f_{2,1}$, which means $f_{1,1}=f_{2,1}$, as $\rho_{1} \neq 0$ Repeating this procedure, we have $f_{1, k}=f_{2, k}, k=1,2, \cdots$. Therefore, we obtain $f_{1}(x)=f_{2}(x), x \in[0, l]$. Analogously, we can prove the case of $\alpha=1$. The proof of the theorem is completed.

\section{VARIATIONAL OPTIMIZATION AND THE CONJUGATE GRADIENT METHOD}

Since the inverse source problem is ill-posed, we consider using the classical Tikhonov regularization method to deal with the problem. Define a Tikhonov regularization functional as follows

$$
J(f)=\frac{1}{2}\left\|K_{\alpha}(f)-g^{\delta}\right\|_{L^{2}(0, l)}+\frac{\beta}{2}\|f\|_{L^{2}(0, l)}
$$

where $\beta$ is a regularization parameter. Therefore, we transform the inverse source problem into the following variational optimization problem to solve

$$
\min _{f \in L^{2}(0, l)} J(f)
$$

In this paper, we use the conjugate gradient method(CGM) to search the minimizer of functional (23). Here we draw the functional gradient with the help of the variational adjoint method.

In the following, we formally give the procedure of deriving the functional gradient. Let $\delta f(x)$ be a small perturbation of the space-dependent source $f(x)$, then the small change $\omega=u[f+\delta f](x, t)-u[f](x, t)$ of the solution to the direct problem (1)-(3) satisfies

$$
\begin{cases}\frac{\partial^{\alpha} \omega(x, t)}{\partial t^{\alpha}}=(L \omega)(x, t)+\delta f(x), & x \in(0, l), t>0,0<\alpha \leq 1, \\ \frac{\partial \omega}{\partial x}(0, t)=\frac{\partial \omega}{\partial x}(l, t)=0, & t \geq 0, \\ \omega(x, 0)=0, & x \in[0, l] .\end{cases}
$$

In order to get the gradient of the optimization functional (23), we firstly compute the first order variation of functional (23). From (23), we have

$\delta J(f)=J(f+\delta f)-J(f)$

$=\frac{1}{2}\left\|K_{\alpha}(f+\delta f)-g^{\delta}\right\|_{L^{2}(0, l)}+\frac{\beta}{2}\|f+\delta f\|_{L^{2}(0, l)}$

$-\frac{1}{2}\left\|K_{\alpha}(f)-g^{\delta}\right\|_{L^{2}(0, l)}-\frac{\beta}{2}\|f\|_{L^{2}(0, l)}$

$=\int_{0}^{T}\left(u[f](0, t)-g^{\delta}(t)\right) \omega(0, t) d t+\beta \int_{0}^{l} f(x) \delta f(x) d x$

Let $v(x, t)$ be a smooth function, multiplying both side of 
equation (25) by $v(x, t)$ and integrating with respect to the temporal-spatial variables on $Q=[0, T] \times[0, l]$, we have

$0=\int_{0}^{l} \int_{0}^{T}\left(\partial_{t}^{\alpha} \omega(x, t)-L(\omega)(x, t)-\delta f(x)\right) v(x, t) d t d x$

$=\int_{0}^{l} \int_{0}^{T} v(x, t) \int_{0}^{l} \frac{1}{\Gamma(1-\alpha)}(t-\tau)^{-\alpha} \frac{\partial \omega(x, \tau)}{\partial \tau} d \tau d t d x$

$-\int_{0}^{l} \int_{0}^{T} L(\omega)(x, t) v(x, t) d t d x$

In order to find the gradient of the functional $J(f)$, we define the adjoint problem as follows

$\begin{cases}\partial_{T_{-}}^{\alpha} v(x, t)=L(v)(x, t), & x \in(0, l), t>0,0<\alpha \leq 1, \\ \frac{\partial v(0, t)}{\partial x}=u[f](0, t)-g^{\delta}(t), \frac{\partial v(l, t)}{\partial x}=0, & t>0, \\ v(x, T)=0, & x \in[0, l] .\end{cases}$

where $\partial_{T_{-}}^{\alpha} y(t)$ is the Caputo fractional right derivative defined by

$\partial_{T_{-}}^{\alpha} y(t)= \begin{cases}-\frac{1}{\Gamma(1-\alpha)} \int_{t}^{T} \frac{y^{\prime}(s)}{(s-t)^{\alpha}} d s, & 0<\alpha<1, \\ -\frac{d y(t)}{d t}, & \alpha=1 .\end{cases}$

We can obtain

$J^{\prime}(f)=\int_{0}^{T} \frac{1}{\Gamma(2-\alpha)} t^{1-\alpha} \partial_{t} v(x, t) d t+\beta f, 0<\alpha \leq 1$.

Assume that $f_{k}(x)$ is the $k-$ th approximate solution of $f(x)$. Set

$f_{k+1}=f_{k}+\vartheta_{k} d_{k}, k=0,1, \cdots$,

where $d_{k}$ is a descent direction and $\vartheta_{k}$ is the step size in the $k$-th iteration. The descent direction of the CGM is updated by using the following iteration formula

$d_{k}=-J^{\prime}\left(f_{k}\right)+\varsigma_{k} d_{k-1}$,

where $\varsigma_{k}$ conjugate coefficient computed by

$\varsigma_{k}=\frac{\int_{0}^{T}\left(J^{\prime}\left(f_{k}\right)\right)^{2} d t}{\int_{0}^{T}\left(J^{\prime}\left(f_{k-1}\right)\right)^{2} d t}, \varsigma_{0}=0$.

we have

$\vartheta_{k}=-\frac{\int_{0}^{T}\left(K_{\alpha}\left(f_{k}\right)-g^{\delta}\right) K_{\alpha}\left(d_{K}\right) d t+\beta \int_{0}^{l} f_{k} d_{k} d x}{\int_{0}^{T}\left(K_{\alpha}\left(d_{k}\right)\right)^{2} d t+\beta \int_{0}^{l} d_{k}^{2} d x}$.

So, we formulate the inversion algorithm based on the CGM to solve the inverse source problem as follows

\begin{tabular}{|c|c|}
\hline Algorithm & $\begin{array}{l}\text { The Conjugate gradient inversion algorithm for the inverse } \\
\text { source problem }\end{array}$ \\
\hline Step 1 & initialize $f_{0}=0$, and set $k=0$; \\
\hline Step 2 & $\begin{array}{l}\text { Solve the direct problem (1)-(3) with } f=f_{k} \text {, and calculate } \\
\text { the residual } e_{k}=K_{\alpha}\left(f_{k}\right)-g^{\delta}(t)\end{array}$ \\
\hline $\begin{array}{l}\text { Step } 3 \\
\text { Step } 4\end{array}$ & $\begin{array}{l}\text { Solve the adjoint problem }(28) \text { to find the gradient } \\
\text { Compute the conjugate coefficient by (32) and the descent } \\
\left.\text { direction } d_{k} \text { by ( } 31\right) \text {; }\end{array}$ \\
\hline Step 5 & Calculate the sensitivity problem (25) with $\delta f_{k}(x)=d_{k}$; \\
\hline Step 6 & Compute the optimal step size $\vartheta_{k}$ by (33); \\
\hline Step 7 & Update the initial value $f_{k+1}$ by (30); \\
\hline Step 8 & $\begin{array}{l}\text { Increase } k \text { by one and return to Step } 2 \text {, repeat the above } \\
\text { process until a stopping criterion is satisfied. }\end{array}$ \\
\hline
\end{tabular}

\section{NUMERICAL EXAMPLES}

To verify the validity of the algorithm, several numerical examples are given in this section. Without loss of generality, we take $l=1$ for all the numerical simulations. In order to obtain (noisy) observation data $g^{\delta}(t)$, we obtain it in the form of a true solution plus perturbation. That add pointwise noise by $g^{\delta}\left(t_{i}\right)=K_{\alpha}\left(f^{*}\right)\left(t_{i}\right)(1+\delta \xi)$, where $f^{*}$ is the true source, $t_{i}$ is the discretization time nodal point, $\delta$ is the noise level and $\xi$ is a uniform random variable in $[-1,1]$. In the iteration procedure, we take the well-known Morozovs discrepancy principle as the stopping condition, i.e., we choose $k$ satisfying the following inequality:

$$
e_{k} \leq \tau \delta \leq e_{k-1}
$$

where $\tau$ is a positive constant.

Example 1. Let $\alpha=0.7, a(x)=1, c(x)=0$. We take $f(x)=\pi^{2} \cos (\pi x)$ as the exact source. The exact solution for the direct problem (1)-(3) is $u(x, t)=\left(1-E_{\alpha, 1}\left(-\pi^{2} t^{\alpha}\right)\right) \cos \pi x$.

Example 2. Let $\alpha=0.8, a(x)=1, c(x)=0$. We take $f(x)=3 x^{2}-2 x^{3}$ as the exact source, and have no exact solution for the direct problem (1)-(3).

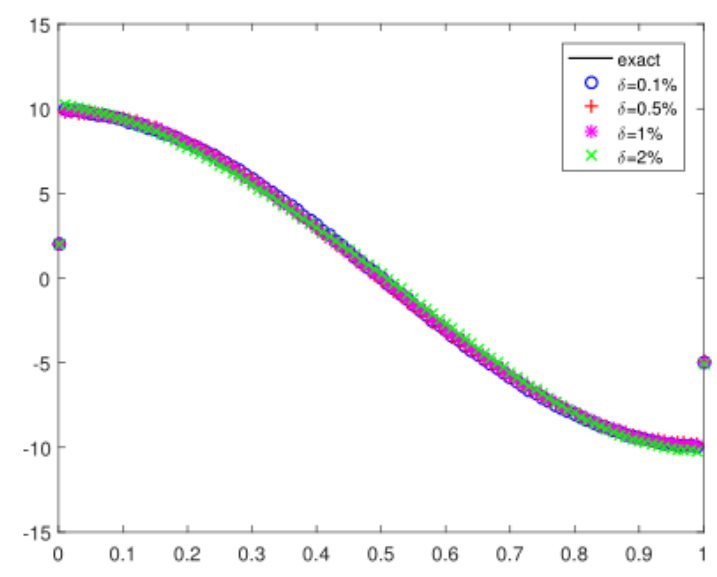

Figure 1: inversion solution for example 1

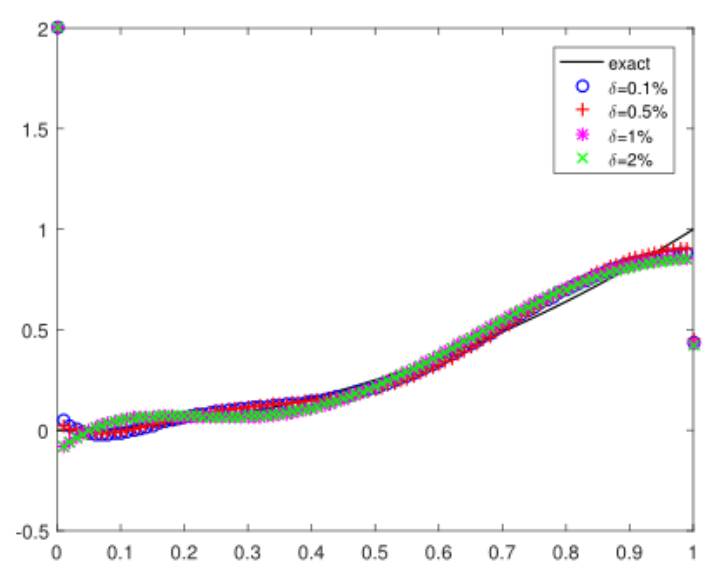

Figure 2: inversion solution for example 2

Example 3. Let $\alpha=1, a(x)=1, c(x)=0$. We take $f(x)=\cos (2 \pi x)$ as the exact source. The exact solution for the direct problem (1)-(3) is $u(x, t)=\left(1-e^{-t}\right) \cos (2 \pi x)$.

Example 4. Let $\alpha=1, a(x)=1, c(x)=0$. We take 
$f(x)=-2 x+\cos (\pi x)^{2}$ as the exact source, and have no exact solution for the direct problem (1)-(3).

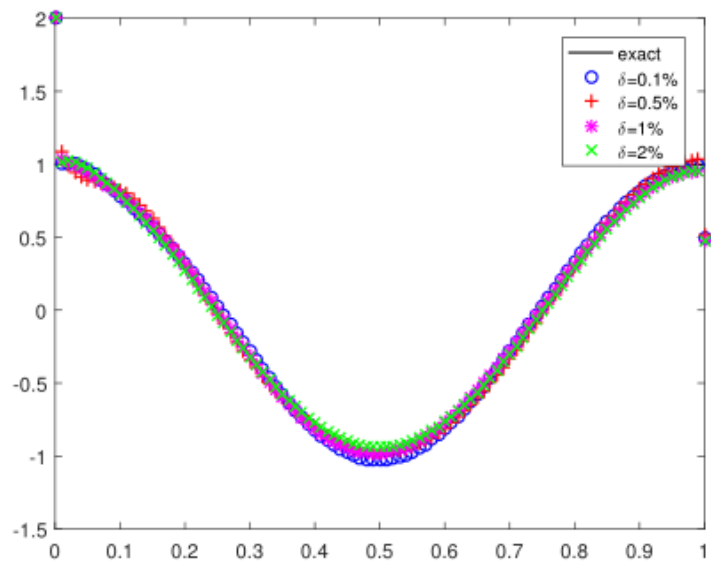

Figure 3: inversion solution for example 3

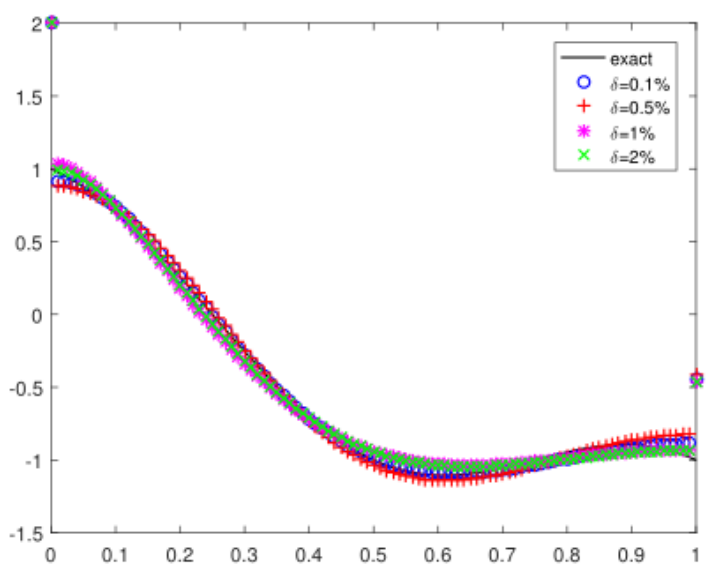

Figure 4: inversion solution for example 4

The above illustration shows the reconstruction of example $1,2,3$ and 4 . The relative noise levels are also chosen as $\delta=0.1 \%, 0.5 \%, 1 \%, 2 \%$, we know the reconstructions are reasonable.

\section{ACKNOWLEDGMENT}

This work is supported by National Natural Science Foun dation of China (11661004,11861007).

\section{REFERENCES}

[1] G.Chi, G.Li, X.Jia, Numerical inversions of a source term in the FADE with a Dirichlet boundary condition using final observations, Comput.Math.Appl. 62(4)(2011)1619-1626.

[2] D.A.Murio, C.E.Mejia, Source terms identification for time fractional diffusion equation, Rev.Colomb.Mat. 42(1)(2008)25-46.

[3] D.A.Murio, Stable numerical solution of a fractional-diffusion inverse heat conduction problem, Comput.Math.Appl. 53(2007)1492-1501.

[4] Y.Zhang, X.Xu, Inverse source problem for a fractional diffusion equation, Inverse Probl. 27(3)(2011)035010.

[5] G.H.Zheng, T.Wei, Spectral regularization method for a Cauchy problem of the time fractional advection-dispersion equation, J.Comput.Appl.Math. 233(10)(2010)2631-2640.

[6] B.Jin, W.Rundell, An inverse problem for a one-dimensional time-fractional diffusion problem, Inverse Probl. 28(7)(2012)19.

[7] X.T.Xiong, X.M.Xue, A fractional Tikhonov regularization method for identifying a space-dependent source in the time-fractional diffusion equation, Applied Mathematics and Computation. 349(2019)292-303.
[8] X.B.Yan, T.Wei, Inverse space-dependent source problem for a time-fractional diffusion equation by an adjoint problem approach. J. Inverse Ill-Posed Probl. 2018; aop.

[9] Z.W.Wang, W.Zhang, B.Wu, Regularized optimization method for determining the space-dependent source in a parabolic equation without iteration. Journal of Computational Analysis and Applications, June 2016.

[10] Z.S.Ruan, J.Z.Yang, X.L.Lu, Tikhonov regularisation method for simultaneous inversion of the source term and initial data in a time-fractional diffusion equation. East Asian Journal on Applied Mathematics, Vol.5, No.3, pp.273-300.

[11] J.G.Wang, T.Wei, Quasi-reversibility method to identify a space-dependent source for the time-fractional diffusion equation, Appl.Math.Model. 39(2015)6139-6149.

[12] F.F.Dou, C.L.Fu, F.Yang, Identifying an unknown source term in a heat equation, Inverse Probl.Sci.Eng. 17(7)(2009)901-913.

[13] J.G.Wang, Y.B.Zhou, T.Wei, A posteriori regularization parameter choice rule for the quasi-boundary value method for the backward time-fractional diffusion problem, Appl.Math.Lett.26(7)(2013)741-747.

[14] J.G.Wang, Y.B.Zhou, T.Wei, Two regularization methods to identify a space-dependent source for the time-fractional diffusion equation, Appl.Numer.Math.68(2013)39-57.

[15] P.Zhuang, F.Liu, Implicit difference approximation for the time fractional diffusion equation. Journal of Applied Mathematics and Computing, 22(3):87-99, 2006.

[16] J.Liu, M.Yamamoto, A backward problem for the time-fractional diffusion equation, Appl.Anal. 89, 1769-1788(2010).

[17] R.Metzler, J.Klafter, Boundary value problems for fractional diffusion equations, Phys. A. 278,107-125(2000).

[18] B.Jin, R.William, An inverse problem for a one-dimensional time-fractional diffusion problem, Inverse Problems. 28,075010(2012).

[19] X.Li, C.Xu, A space-time spectral method for the time-fractional diffusion equation, SIAMJ. Num. Anal. 47, 2108-2131(2009).

[20] H.Pollard, The completely monotonic character of the Mittag-Leffler function $E_{\alpha}(-x)$. Bulletin of the American Mathematical Society, 1948, 54(12): 1115-1116.

[21] I.Podlubny, Fractional differential equations: an introduction to fractional derivatives, fractional differential equations, to methods of their solution and some of their applications, Mathematics in science and engineering, 198, San Diego, CA: Academic Press Inc; 1999

[22] Levitan B M, Sargsian I S, Sargsjan I S. Introduction to spectral theory: selfadjoint ordinary differential operators: Selfadjoint Ordinary Differential Operators. American Mathematical Soc., 1975. 